\title{
CUSTOS DOS SERVIÇOS DE TERCEIROS VERSUS SERVIDOR EFETIVO NA GESTÃO PÚBLICA MUNICIPAL
}

COSTS OF THIRD PARTY SERVICES

VERSUS EFFECTIVE SERVER IN MUNICIPAL PUBLIC MANAGEMENT

LUÍS FERNANDO PEIXOTO WESZ

Universidade Regional Integrada - URI

$B R A S I L$

ROBERTO CARLOS DALONGARO

Universidade Regional Integrada - URI

BRASIL

La llave del éxito es arriesgarse a tener pensamientos no convencionales. La convención es el enemigo del progreso.

Trevor Baylis 


\section{RESUMO}

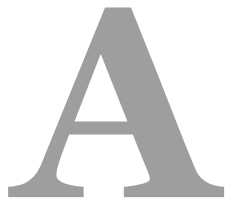

tualmente a gestão do setor público prioriza o controle dos recursos e dos procedimentos formais, e não os resultados, de desempenho, a eficiência, por esta razão se faz relevante identificar a despesa e custo, correspondentes aos desembolsos financeiros dos atos desenvolvidos pela gestão dos governos. Neste contexto identifica-se que as despesas mostram como o governo financia os recursos que usa, enquanto os custos mostram como o governo financia seus resultados. Para tanto, a intensificação da crise fiscal no estado brasileiro, e na economia nacional e internacional, recomenda que o Estado tenha uma nova orientação política e econômica, em especial na contenção de suas despesas. Portanto, é oportuno se inserir no gasto público, a aplicabilidade e qualidade dos gastos de governo. O presente artigo busca identificar a importância em apurar os custos no serviço público e a partir desta justificativa a necessária apuração destes. Em seguida o trabalho pesquisa os custos de implantação de uma oficina mecânica junto a gestão pública municipal e a contratação de um servidor para o desempenho desta atividade. Por fim, busca identificar o custo benefício do Serviço Terceirizado versus Servidor Concursado e seus custos na gestão pública municipal.

Palavras Chave: gestão pública, custos, prestação de serviços.

\section{ABSTRACT}

Currently the public sector management prioritizes the control of resources and formal procedures, not results, performance, efficiency, therefore, it is important to identify the expenditure and cost, corresponding to the financial expenses of acts developed by The management of government. In this context, it is identified that expenditures show how the government finances the resources it uses, while costs show how the government finances its results. Therefore, the intensification of the fiscal crisis in the Brazilian state and in the national and international economy, recommends that the State has a new political and economic direction, in particular in the containment of expenses. Therefore, it is appropriate to incorporate in the public expenditure, the applicability and the quality of the public expenditure. This article seeks to identify the importance of determining the costs in the public service and from there the necessary justification for the verification of these. The work then investigates the costs of deploying a mechanical workshop together with the municipal management and the hiring of a employee to carry out this activity. Finally, it seeks to identify the cost benefit of the outsourced service vis-à-vis the bankrupt employee and its costs in the municipal public administration.

Keywords: public administration, costs, provision of services. 


\section{RESUMEN}

Actualmente la gestión del sector público prioriza el control de los recursos y los procedimientos formales, y no los resultados, el rendimiento, la eficiencia, por lo tanto, es importante identificar el gasto y el costo, correspondiente a los gastos financieros de los actos desarrollados por la gestión de gobierno. En este contexto se identifica que los gastos muestran cómo el gobierno financia los recursos que utiliza, mientras que los costos muestran cómo el gobierno financia sus resultados. Por lo tanto, la intensificación de la crisis fiscal en el estado brasileño y en la economía nacional e internacional, recomienda que el Estado tenga una nueva dirección política y económica, en particular en la contención de los gastos. Por lo tanto, es oportuno incorporar en el gasto público, la aplicabilidad y la calidad del gasto público. Este artículo busca identificar la importancia de determinar los costos en el servicio público y a partir de ahí la justificación necesaria para la verificación de estos. A continuación, el trabajo investiga los costes de despliegue de un taller mecánico junto a la gestión municipal y la contratación de un empleado para llevar a cabo esta actividad. Por fin, busca identificar el costo beneficio del servicio tercerizado frente al servidor efectivo y sus costos en la administración pública municipal.

Palabras clave: administración pública, costos, prestación de servicios.

\section{INTRODUÇÃO}

Atualmente ejá em décadas passadas como 70 e 80 tem se intensificado a crise fiscal no estado brasileiro, em contrapartida destas dificuldades uma maior exposição da economia nacional a competição internacional, em razão destas alterações se faz necessário que o Estado tenha uma nova orientação a sua política econômica, em especial na contenção de suas despesas. Em razão da dificuldade em aumentar a carga tributária, a palavra de oportunidade passou a ser cortar gastos. No entanto, a atual circunstância macroeconômica vem reafirmando ao mundo a necessidade de uma gestão Pública que valorize a qualidade do gasto público, e não simplesmente pelo corte do gasto público. As despesas mostram como o governo financia os recursos que usa - despesa com pessoal, despesa com material de consumo, despesa com equipamento e material permanente, despesa com serviços de terceiros, despesas financeiras, etc. - enquanto os custos mostram como o governo financia seus resultados - custo de uma aula, custo de uma consulta médica, custo de um serviço administrativo, custo de uma operação de fiscalização, etc.

Atualmente o modelo de gestão do setor público prioriza o controle dos recursos e dos procedimentos formais, e não os resultados, o desempenho, a eficiência. Sendo assim a razão principal pelo conhecimento de custos no serviço público, ser de relevante 
importância para a alocação eficiente dos recursos. Enfim, Custos no serviço público demonstra ser de extrema importância para a Gestão da Administração Pública.

Este trabalho resulta da realização de um estudo de Caso, utilizando os métodos qualitativos e quantitativos, apoiado pelos aportes teóricos sobre os conceitos Custos no Serviço Público, seguido de uma pesquisa exploratória em relatório de Auditoria do Controle Interno Municipal, através da análise de dados, visando à obtenção de informações sobre o problema em tela. A pesquisa teve como foco amostral a organização da Administração Direta, vinculadas ao Poder Executivo Municipal, que buscam de alguma maneira a melhoria em seus projetos e ações relacionados à melhoria da qualidade dos produtos e serviços que fornecem e/ou dos processos internos necessários para sua realização, bem como estejam utilizando ou desenvolvendo práticas de gestão relacionadas a sistemas de custeio e contabilidade de custos.

Para contextualizar a respectiva problemática de estudo, é levantado o seguinte questionamento: Qual a importância em apurar os custos no serviço público municipal?

\section{ASPECTOS CONCEITUAIS}

Os custos, somente apresentam sentido se vinculados a sistemas de avaliação de desempenho institucional. Tais avaliação de desempenho, pressupõe um planejamento estratégico e de gestão de processos. O bom desempenho depende da utilização racional dos recursos, priorizadas no planejamento estratégico.

Para Alonso (1996), o planejamento estratégico define os objetivos estratégicos, o que a organização quer atingir, e os macroprocessos, que são as grandes funções da organização, consistentes com a missão e a visão de futuro da organização. O detalhamento operacional dos objetivos estratégicos produz a definição das metas para um determinado período, ao passo que o desdobramento dos macroprocessos resulta na definição dos processos de trabalho.

Segundo Silveira (2006), com o custeamento de todos os processos de trabalho da Instituição, inclusive os processos interdepartamentais, os gestores contarão com uma visão mais holística da organização, sem as "amarras" da tradicional abordagem hierárquica, que privilegia a análise da organização em função do organograma e não dos processos da atividade. Essa Compatibilização entre o Sistema de Custos e os reais processos de trabalho das unidades permitirá uma visão completa por processos. No mapeamento dos processos já existentes, procede-se à uma descrição razoavelmente detalhada das atividades que compõe tais processos de trabalho. Este mapa de processos e a lista de atividades são a matéria-prima para os modernos sistemas de custos.

Portanto, a compreensão sobre o planejamento estratégico e os macroprocessos são componentes essenciais na identificação do método de custo que melhor se adapta a Gestão 
de Custos no Serviço Público, atuando como ferramenta primordial para a visibilidade das atividades que geram custos, neste contexto, destaca-se o método de Custeio $\mathrm{ABC}$, com sua metodologia os processos de mudança organizacional e os programas de qualidade têm sido destacados universalmente através da literatura especializada: que o melhor local para controlar custos é no nível dos processos (Alonso, 1998).

Entretanto,Alonso(1997) esclareceque, quando se depende de um sistema decontabilidade orçamentária e de verbas, muitas decisões financeiras são tomadas arbitrariamente. Ao invés de reduções de custos seletivas, no qual, ocorrem reduções (lineares horizontais). Na maioria dos casos, isto acontece simplesmente porque os gerentes de nível superior não têm ideia de que partes do processo agregam mais valor aos produtos e serviços e quais as que agregam menos, enquanto os funcionários no nível dos processos são capazes de determinar este valor, mas precisam ter um indicador do custo real. O custo baseado em atividade poderá fornecer esse tipo de informação. Por fim, destaca-se que este método é amplamente utilizado nos EUA, sendo uma metodologia reconhecidamente eficaz.

O destaque pela escolha do método de Custeio Baseado em Atividades (ABC), se deu pelo fato de que este possibilita a alocação de despesas administrativas aos produtos e serviços e permite a mensuração dos custos que não agregam valor, contribuindo para a melhor análise dos processos de trabalho, eliminando desperdícios e otimizando o uso dos recursos orçamentários. Sua principal característica, quando comparada com os demais métodos tradicionais de apuração de custos, consiste no tratamento refinado dos custos indiretos (Martins, 1998), daí sua adoção significativa por parte de empresas prestadoras de serviços e instituições públicas.

\section{1 É necessário apurar custos no serviço público municipal?}

Urge a necessidades em apurar os custos dos serviços públicos, através de uma reforma que melhore a qualidade do gasto público. As preocupações na gestão de custos deverão estar relacionadas com o desempenho dos serviços públicos. Visto que, a melhora no desempenho de uma Instituição Governamental, requer sistemas de informações gerenciais que deem suporte consistente aos seus processos decisórios da gestão (Kaplan Norton, 1997).

Porém, com relação à medição de custos a situação é ainda mais precária, pois não há tradição em se medir custos no serviço público. Por exemplo, quanto custa um aluno? Um determinado serviço de saúde? Os serviços jurídicos? Quais atividades agregam valor nos processos de trabalho nas Instituições governamentais? Qual é o impacto em termos de custos nas mudanças organizacionais propostas ou implementadas? Qual é a eficiência de cada órgão e ou Secretaria de governo? Por essas razões a Sociedade não sabe quanto custa os serviços públicos. Não havendo medida de custos, também não há medida de eficiência, dado que a eficiência é a relação entre resultados e o custo de obtê-los (Kaplan; Cooper, 1998). 
A constatação da experiência nacional e internacional tem demonstrado que a apuração e a divulgação de medidas de desempenho constituem um poderoso instrumento nos processos de mudança organizacional. Assim, a apuração e a divulgação dos indicadores de resultado atuam como um guia para a mudança organizacional, tais medidas de desempenho prestamse, para os seguintes objetivos: Promover a redução de custos com melhoria na qualidade dos serviços; instrumentalizar e combate ao desperdício; tornar claro a percepção de melhora no desempenho dos serviços prestados; Incentivar a mudança da cultura organizacional; Subsidiar a formação do processo orçamentário; Subsidiar a avaliação nos planos de reestruturação e melhoria da gestão pública (Nakagawa, 1993).

Portanto nos dias atuais é oportuno que as Instituições Públicas procurem adotar sistemas de custos, por ser esta condição, necessária para melhora na qualidade dos serviços prestados, pois a Administração Pública torna-se mais eficiente quando consegue oferecer os mesmos serviços, em quantidade e qualidade, a custos menores.

\subsection{Custos serviços e instalação}

\subsubsection{Prestação de serviços de terceiros}

A Secretaria de Obras e de Agricultura em destaque neste artigo são unidades integrantes da Gestão Municipal com atuação no município de Caibate RS. No setor público um dos critérios mais relevante é identificar se os custos estão elevados em relação a padrões de análise os quais poderão orientar a Administração Pública em ações de melhoria ou de reestruturação de processos. A adequada apuração dos custos dos serviços públicos é um poderoso instrumento de controle social, permitindo aos usuários, a avaliação da eficiência dos serviços prestados. Nos dias atuais, apenas "saber muito" sobre alguma coisa não proporciona, maior poder de competição para uma organização. Hoje a Gestão do Conhecimento contribui no processo sistemático de identificação, criação, renovação e aplicação dos conhecimentos que são estratégicos na vida de das Instituições. Permitindo às organizações a mensurar com mais segurança e eficiência, tomando decisões mais adequadas, sabendo administrar dados e informações e a gerenciar seus conhecimentos (Sveiby, 1998).

\section{ASPECTOS METODOLÓGICOS E ANÁLISE DOS RESULTADOS}

\subsection{Tipologia da pesquisa quanto aos procedimentos}

Segundo Gil (2002), a classificação das pesquisas em exploratórias, descritivas e explicativas é muito útil para o estabelecimento de seu marco teórico, ou seja, para possibilitar 
uma aproximação conceituai. Todavia, para analisar os fatos do ponto de vista empírico, para confrontar a visão teórica com os dados da realidade, torna-se necessário traçar um modelo conceituai e operativo da pesquisa.

Então quanto aos seus procedimentos a pesquisa se enquadra com bibliográfica e documental.

Segundo o respectivo autor citado anteriormente, a pesquisa bibliográfica é desenvolvida com base em material já elaborado, constituído principalmente de livros e artigos científicos.

Embora em quase todos os estudos seja exigido algum tipo de trabalho dessa natureza, há pesquisas desenvolvidas exclusivamente a partir de fontes bibliográficas. Boa parte dos estudos exploratórios pode ser definida como pesquisas bibliográficas, dessa forma caracterizase o procedimento de pesquisa bibliográfica para o presente estudo em desenvolvimento, em função dos diversos conceitos teóricos tecidos ao longo do referencial bibliográfico constituído no capítulo 2 deste trabalho.

Já a pesquisa documental, segundo Gil (2002), assemelha-se muito à pesquisa bibliográfica. A diferença essencial entre ambas está na natureza das fontes. Enquanto a pesquisa bibliográfica se utiliza fundamentalmente das contribuições dos diversos autores sobre determinado assunto, a pesquisa documental vale-se de materiais que não recebem ainda um tratamento analítico, ou que ainda podem ser reelaborados de acordo com os objetos da pesquisa.

Para o referido autor, o desenvolvimento da pesquisa documental segue os mesmos passos da pesquisa bibliográfica. Apenas cabe considerar que, enquanto na pesquisa bibliográfica as fontes são constituídas sobretudo por material impresso localizado nas bibliotecas, na pesquisa documental, as fontes são muito mais diversificadas e dispersas. Há, de um lado, os documentos "de primeira mão", que não receberam nenhum tratamento analítico.

\subsection{Tipologia de pesquisa quanto abordagem}

A pesquisa será predominantemente quantitativa, pelo fato de apresentar números absolutos e em percentuais relacionados aos custos de produção levantados, como também, números absolutos em relação aos valores dos custos e a quantidade unitária de produção envolvida no processo de produção a ser pesquisado.

Segundo Roesch (2015), a pesquisa quantitativa é apropriada para avaliar mudanças em organizações. Quando se trata de programas abrangentes, com resultados do trabalho, sistema participativo, que acompanha a evolução dos acontecimentos de forma continua. Também explora dados numéricos, relacionados a percentuais e números absolutos que mostram a realidade do cenário pesquisado.

A pesquisa também abordara aspectos qualitativos, em função da necessidade de se embasar teoricamente os conceitos relacionados aos custos, as características dos custos, a formação do serviço prestado, entre outros constructos teóricos relevantes para o desenvolvimento conceitual do presente trabalho de pesquisa. 
Argumenta-se que a pesquisa qualitativa e seus métodos de coleta e análise de dados são apropriados para a fase exploratória da pesquisa. A pesquisa qualitativa também é apropriada para a avaliação formativa, quando se trata de melhorar a efetividade de um programa, ou plano, e também quando se trata de relatar uma evolução histórica de determinado tema e construir considerações críticas sobre o seu aspecto evolutivo (Roesch, 2015).

\subsection{O estudo de caso proposto}

O Estudo de caso foi pautado no Relatório Simplificado $\mathrm{n}^{0}$ : 01/2017 realizado auditoria no sistema Custos de Serviços na Secretaria Obras e Agricultura no período compreendido entre 2013 a 2016. A auditoria procedeu com as seguintes etapas: a) Pesquisa relatórios de Serviços período de 2013 a 2016; b) Demonstrativo de cálculos na formação salarial para custear um mecânico no quadro municipal; c) Formação de Custos para instalação de uma oficina mecânica; d) Análise Custo Benefício Contratação Mecânico versus Contração Serviços direto nas empresas.

Neste levantamento de informações, identificou-se o montante que foi gasto no período em observação, o qual correspondeu a $\mathrm{R} \$ 414.327,72$ (quatrocentos e quatorze mil, trezentos e vinte e sete reais e setenta e dois centavos), e os acréscimos de aumentos nos respectivos anos com base no ano de 2013 até 2016. Representados da seguinte forma, no ano de 2014 houve um aumento na despesa em um percentual de 35\% (a maior), com relação às despesas de 2015, houve um aumento de 79\% e em 2016 um aumento de 93\% ambos em comparação ao ano base de 2013.

\subsection{Demonstrativo formação salarial servidor público - mecânico}

Nesta observação compreende a composição da remuneração de um Servidor ao qual foi identificado existir a vaga no quadro funcional da Prefeitura Municipal. Estando classificada a remuneração do Mecânico no padrão 06 o qual equivale 4,25 do Padrão de referencial salarial (PRS) acrescido de 20\% de Insalubridade, compondo assim a remuneração do Servidor. Quanto aos benefícios identifica-se, o vale alimentação.

Com relação aos Insumos identificou os equipamentos que devem ser utilizados, como botas, macacão, óculos e demais utensílios necessários ao desenvolvimento de suas atividades nas questões de segurança no trabalho. Por fim demonstra os Encargos incidentes sobre a remuneração. Por fim compreende-se serem estes quesitos os formadores da remuneração do Servidor. Nesta análise foi demonstrado o valor do custo mensal, equivalente a $\mathrm{R} \$$ 2.506,0o (dois mil, quinhentos e seis reais) aos cofres Públicos e também o custo anual o qual corresponde ao valor de $\mathrm{R} \$ 30.071,94$ (trinta mil, setenta e um reais e noventa e quatro centavos), enquanto no período de quatro anos correspondeu a $\mathrm{R} \$ 120.287,76$ (cento e vinte mil, duzentos e oitenta e sete reais e setenta e seis centavos). 


\subsection{Qual o custo de instalação de uma oficina mecânica na prefeitura municipal de Caibaté?}

O Investimento necessário para a formação de uma oficina de aproximadamente 200 $\mathrm{m}^{2}$ e capacidade para acomodar 1 veículo em elevador automotivo, 1 veículo na troca de óleo, 1 veículo no alinhamento e outros 2 veículos em Box de atendimento, o a Prefeitura deverá dispor de aproximadamente $\mathrm{R} \$ 115.000,00$, (Fonte pesquisa SEBRAE).

\subsection{Qual o custo benefício na proposta de implantação de uma oficina mecânica na prefeitura municipal de Caibaté?}

Tabela 1. Análise Custos x Benefícios Serviços Manutenção Veículos

\begin{tabular}{|l|c|c|}
\hline Descrição fatos & Valores R\$ & $\%$ \\
\hline 1. Serviços terceirizados em 4 anos & $\mathrm{R} \$ 414.327,72$ & $100 \%$ \\
\hline 2. Custo Servidor em 4 anos & $\mathrm{R} \$ 120.287,76$ & $29,03 \%$ \\
\hline 3. ECONOMIA (1-2) & $\mathrm{R} \$ 294.039,94$ & $70,96 \%$ \\
\hline 4.Custo Instalação Oficina & $\mathrm{R} \$ 115.000,00$ & $27,75 \%$ \\
\hline (=) Resultado (3-4) & $\mathrm{R} \$ 179.039,94$ & $43,21 \%$ \\
\hline
\end{tabular}

Fonte: Elaborado pelos autores

Os custos analisados no presente estudo foram demonstrados a partir dos custos dos serviços terceirizados nos quatros anos (de 2013 a 2016), os quais representaram um montante de $\mathrm{R}$ \$14.327,72 e pelo Custo de remuneração de um Servidor Mecânico, representado pelo montante de $\mathrm{R} \$ 120.287,76$. Neste estudo comparativo dos dois custos pode-se constatar a possibilidade de gerar uma Economia aos cofres públicos na ordem de R \$ 294.039,94.

A partir desta constatação foi identificado que os benefícios que poderão ser propiciados a Gestão Pública com a instalação de uma oficina, visto que, se oportunizou uma economia de $\mathrm{R} \$ 294.039,94$, o que tornaria possível um investimento de aproximadamente $\mathrm{R} \$$ 115.000,00 o qual poderá ser diluído ao longo dos anos e enfatizando que seria possível obter um resultado (sobras) de $\mathrm{R} \$ 179.039,94$, e este por sua vez poderia ser investido outras demandas municipais. Ressalta-se que o estudo fora realizado em um período de quatro anos, então ao optar-se por esta nova possibilidade pode ter o município significativa Economia, gerindo assim os recursos públicos com maior eficiência e eficácia. Face o exposto, foram as seguintes as recomendações ao gestor municipal: a) Possibilidade em chamar via concurso um mecânico; b) Investimento em equipamentos e formação de uma oficina mecânica junto ao parque de máquinas no município; c) Considerar o estudo de viabilidade dos Custos apurados para tomada de decisão administrativa. 


\section{CONCLUSÃo}

Nos tempos atuais só faz sentido estabelecer parcerias quando o custo das atividades é menor que o praticado pela Administração Pública, até porque as parcerias quase sempre utilizam recursos públicos, e estes estão sujeitos ao (novo) princípio constitucional da eficiência. Ou seja, a concepção moderna de parceria pressupõe uma adequada gestão de custos.

A melhoria no desempenho de uma organização governamental requer a utilização de sistemas de custos e informações gerenciais que deem sustentação aos processos decisórios. Preconiza a Lei de Responsabilidade Fiscal Lei Complementar 101, e estabelece que as organizações públicas devam se pautar por critérios de economicidade, eficiência, eficácia e, para isso, devem definir métodos de custeamento. Além disso, outro fator decisivo para se decidir pela implantação de custos na gestão pública, foi à possibilidade de utilização das informações a serem geradas pelo sistema no aperfeiçoamento dos seus processos de trabalho e das ferramentas de gestão, levando em conta os princípios gerenciais da efetividade, eficácia e eficiência.

Destaca-se de modo significativo alguns objetivos que poderão fazer a diferença no dia a dia das Instituições os quais se apresentam a seguir: mensurar e controlar os custos administrativos na Gestão Pública Municipal; quantificar o valor dos serviços oferecidos à sociedade (macroprocessos); mensurar o grau de eficiência e eficácia no desempenho de suas funções; subsidiar o planejamento da entidade e auxiliar no controle da sua execução e, atender às exigências da LRF. ${ }^{1}$

As análises apresentadas neste artigo indicam que os aperfeiçoamentos que aumentem a utilização gerencial de cálculos de custo e do Sistema de Custos e sua aderência a outros sistemas de controle e gestão não são apenas possíveis como necessários. O Exemplo das Secretarias em questão demonstra mostra que esse enfoque gerencial é possível, indo além da "simples" gestão de custos, integrando e uniformizando diversos instrumentos de controle de forma a subsidiar a tomada de decisão e torná-la mais eficaz. Hoje estas duas Secretarias mesmo que se utilizado do processo manual demonstrou que é possível, desejável e necessário empreender esforços para que todas as Secretarias sejam inseridas nos esforços adequação aos custos reais de seus serviços refletidas no Sistema de Custos e suas redes de processos, além de suas estruturas administrativas. Para finalizar, pode-se afirmar que os sistemas de custos na administração pública estão mais suscetíveis ao sucesso se conseguirem ir além da apropriação e gestão de custos, buscando se atrelarem a outros modelos de gestão e instrumentos de controle, de forma a propiciar uma análise completa das funções, processos, atividades e riscos, o que certamente agregará valor à tomada de decisão dos gestores públicos.

${ }^{1}$ Lei de Responsabilidade Fiscal. 


\section{REFERÊNCIAS BIBLIOGRÁFICAS}

Alonso, Marcos. (1996). “Contabilidade Pública Gerencial”. Escola Nacional de Administração Pública. Brasília - DF. ENAP.

Alonso, Marcos. (1998). "Por um sistema de custos para o MARE”. Nota técnica No o5, documento interno do MARE.

Alonso, Marcos. (1997). “Agências Executivas: estratégias de reforma para a administração indireta”. Escola Nacional de Administração Pública. Brasília - DF. № 18, ENAP.

Carr, D.; Littman, L. (1992). “Excelência nos serviços públicos”. Rio de Janeiro. Qualitymark.

CONSELHO FEDERAL DE CONTABILIDADE. (1995). “Custo como ferramenta gerencial”. São Paulo. Atlas.

Gil, Antonio Carlos. (2002). "Como elaborar projetos de pesquisa”. 4. ed. São Paulo: Atlas.

Kaplan, R.; Cooper, R. (1998). “Custo e Desempenho”. São Paulo: Futura.

Kaplan, R. e Norton, D. (1997). “A estratégia em ação”. Balanced Scorecard. São Paulo: Campus.

Kehoe, J. et all. (1995). “Activity-based management”. Washington, D.C. Coopers \& Librand.

Martins, Eliseu (2010). “Contabilidade de Custos”. São Paulo: Atlas.

Martins, E. (1998). “Contabilidade de custos”. 6. ed. São Paulo: Atlas.

Mcclelland, D. C. (1973). "Testing for Competence rather than Intelligence". American Psychologist. Washington, D.C. 28, 1- 4.

Nakagawa, M. (1994). “ABC: custeio baseado em atividade”. São Paulo: Atlas.

Nakagawa, M. (1993). “Gestão Estratégica de Custos”. São Paulo: Atlas.

Silveira, H.F. R. (2006). “Gestão de custos na administração pública”. In: XI Congreso Internacional del CLAD sobre la Reforma del Estado y de la Administración Pública. Cidade da Guatemala. 
Roesch Sylvia Maria Azevedo. (2015). "Projetos de estágio e de pesquisa em administração: estágios, tcc, dissertações e estudos de caso”. 1. ed. São Paulo: Atlas.

Sveiby, K. E. (1998). “A nova riqueza das organizações”. Rio de Janeiro: Campus.

\section{CURRICULUM VITAE}

\section{Luís Fernando Peixoto Wesz}

Doutorando em Administração UNaM. Mestrado em Agronegócios - UFRGS. Graduado em Ciências Contábeis. Professor de Ciências Contábeis e Administração - URI - São Luiz Gonzaga - Brasil.

l-fernando-wesz@bol.com.br

\section{Roberto Carlos Dalongaro}

Doutorando em Administração - UNaM. Mestrado em Gestão - URI. Graduado em Administração. Professor de Administração e Ciências Contábeis - URI - São Luiz Gonzaga - Brasil. robertocarlosad@hotmail.com 